\title{
EXTINCTION PROBABILITIES OF BRANCHING PROCESSES WITH COUNTABLY INFINITELY MANY TYPES
}

\author{
S. HAUTPHENNE, ${ }^{*}$ The University of Melbourne \\ G. LATOUCHE, ${ }^{* *}$ Université libre de Bruxelles \\ G. NGUYEN, ${ }^{* * *}$ The University of Adelaide
}

\begin{abstract}
We present two iterative methods for computing the global and partial extinction probability vectors for Galton-Watson processes with countably infinitely many types. The probabilistic interpretation of these methods involves truncated Galton-Watson processes with finite sets of types and modified progeny generating functions. In addition, we discuss the connection of the convergence norm of the mean progeny matrix with extinction criteria. Finally, we give a sufficient condition for a population to become extinct almost surely even though its population size explodes on the average, which is impossible in a branching process with finitely many types. We conclude with some numerical illustrations for our algorithmic methods.
\end{abstract}

Keywords: Multitype branching process; extinction probability; extinction criteria; iterative method

2010 Mathematics Subject Classification: Primary 60J80

Secondary 60J05; 60J22; 65H10

\section{Introduction}

Branching processes are powerful mathematical tools frequently used to study the evolution of collections of individuals over time. In particular, multitype Galton-Watson processes represent populations in which individuals are classified into different categories and live for one unit of time. Each individual may reproduce at the end of its lifetime, with reproduction rules dependent on its type.

When the number of types is finite, one extinction criterion is based on the spectral radius $\operatorname{sp}(M)$ of the mean progeny matrix $M$, the elements $M_{i j}$ of which are the expected number of direct offspring with type $j$ for a parent of type $i$. Moreover, the extinction probability vector $\boldsymbol{q}$ is the minimal nonnegative solution of the fixed-point equation $\boldsymbol{q}=\boldsymbol{P}(\boldsymbol{q})$, where each component $q_{i}$ is the extinction probability given the initial type $i$, and $\boldsymbol{P}(\cdot)$ is the progeny generating function of the process. Harris [3] (see also the references therein) presented a comprehensive analysis of extinction criteria and the extinction probability for Galton-Watson processes with finitely many types.

\footnotetext{
Received 16 October 2012; revision received 28 January 2013.

* Postal address: Department of Mathematics and Statistics, The University of Melbourne, VIC 3010, Australia.

Email address: sophiemh@unimelb.edu.au

** Postal address: Département d'Informatique, Université libre de Bruxelles, 1050 Brussels, Belgium.

Email address: latouche@ulb.ac.be

*** Postal address: School of Mathematical Sciences, The University of Adelaide, South Australia 5005, Australia.

Email address: giang.nguyen@ adelaide.edu.au
} 
Allowing, as we do here, the set of types to be infinite gives rise to three main challenges. First, as the mean progeny matrix $M$ has infinite dimension, we have to look for a replacement to the spectral radius as an extinction criterion. Second, we need to determine how to compute the extinction probability vector $\boldsymbol{q}$ which now has infinitely many entries. Third, the concept of extinction has to be defined carefully: when there are infinitely many types, it is possible for every type to eventually disappear while the whole population itself explodes. We use the term global extinction to indicate that the whole population becomes extinct, and represent by $\boldsymbol{q}$ the probability vector for this event; we refer to the event that every type becomes extinct as partial extinction, and denote its probability vector by $\tilde{\boldsymbol{q}}$, with $\boldsymbol{q} \leq \tilde{\boldsymbol{q}}$, naturally, and we ask the question whether they are equal or not.

Galton-Watson processes with infinitely many types have been much investigated already. Moyal [10] assumed that the types belong to an abstract space and proved that the extinction probability is a solution of the fixed-point equation $\boldsymbol{s}=\boldsymbol{P}(\boldsymbol{s})$. Mode [7, Theorem 7.2], for a restricted family of progeny densities, gave an extinction criterion based on the spectral radius of an integral operator. Focusing on denumerably infinite sets of types, Moy [8], [9] and Spataru [14] used ergodic properties for infinite matrices, and analysed in special cases the role of the convergence norm of $M$ as an extinction criterion; Sagitov [12] studied properties of Galton-Watson branching processes with countably many types where the reproduction distributions are linear fractional. Recently, some authors in the literature of branching random walks have defined local survival, meaning that, for every given type $i$ and arbitrarily large epoch $T$, there is at least one individual of type $i$ alive at some time $t>T$, with global survival, meaning that at least one individual is alive at any time, and strong local survival, when the two have the same probability. We refer the reader to [1], [2], and [17].

To date however a simple extinction criteria for general Galton-Watson processes with countably infinitely many types has yet to be found, and the question of actually computing the extinction probability vector has received scant attention, if any.

Our main result is the development of two algorithmic methods for computing the global and the local extinction probability vectors $\boldsymbol{q}$ and $\tilde{\boldsymbol{q}}$. The methods, which are presented in Section 3, have a physical interpretation based on two truncated Galton-Watson processes with finite sets of types. They may be applied to both irreducible and reducible branching processes with countably infinitely many types.

In Section 4 we discuss some extinction criteria expressed in terms of the convergence norm of the mean progeny matrix $M$ when $M$ is irreducible, and of irreducible sub-matrices of $M$ when $M$ is reducible. We also give a sufficient condition under which the population becomes extinct almost surely (a.s.) while its expected size tends to $\infty$. That condition implies that the asymptotic growth rate of the process may depend on the distribution of the initial individual's type.

In Section 5, we provide some numerical illustrations. Our examples are taken from two classes of processes: the matrix $M$ is tridiagonal (and irreducible) or superdiagonal (and reducible).

\section{Preliminaries}

Consider the process $\left\{\mathcal{Z}_{n}=\left(Z_{n 1}, Z_{n 2}, \ldots\right)\right\}_{n \in \mathbb{N}}$, where $Z_{n \ell}$ is the number of individuals of type $\ell$ alive at the $n$th generation, for $\ell$ in the countably infinite set of types $\delta=\{1,2,3, \ldots\}$. Unless otherwise stated, the process starts in generation 0 with one individual.

We denote by $p_{i j}$ for $\boldsymbol{j}=\left(j_{1}, j_{2}, \ldots\right)$ the probability that an individual of type $i$ gives birth to $j_{1}$ children of type $1, j_{2}$ children of type 2 , etc., and the progeny generating function $P_{i}(s)$ 
of type $i \in \delta$ is given by

$$
P_{i}(\boldsymbol{s})=\sum_{\boldsymbol{j} \in \mathbb{N}^{\infty}} p_{i j} \boldsymbol{s}^{j}=\sum_{\boldsymbol{j} \in \mathbb{N}^{\infty}} p_{i j} \prod_{k=1}^{\infty} s_{k}^{j_{k}},
$$

with $s=\left(s_{1}, s_{2}, \ldots\right), s_{i} \in[0,1]$ for all $i$. We define $\boldsymbol{P}(\boldsymbol{s})=\left(P_{1}(\boldsymbol{s}), P_{2}(\boldsymbol{s}), \ldots\right)$. The mean progeny matrix $M$ is defined by

$$
M_{i j}=\left.\frac{\partial P_{i}(s)}{\partial s_{j}}\right|_{s=1} \quad \text { for } i, j \in \&,
$$

and $M_{i j}$ is the expected number of direct offspring of type $j$ born to a parent of type $i$. The process $\left\{\mathcal{Z}_{n}\right\}$ is said to be irreducible if $M$ is irreducible, and it is reducible otherwise.

The total population size at the $n$th generation is $\left|Z_{n}\right|=\sum_{\ell=1}^{\infty} Z_{n \ell}$, and we denote by $\varphi_{0}$ the type of the first individual in generation 0 . The conditional global extinction probability vector, given the initial type, is $\boldsymbol{q}=\left(q_{1}, q_{2}, \ldots\right)$, where

$$
q_{i}=\mathbb{P}\left[\lim _{n \rightarrow \infty}\left|Z_{n}\right|=0 \mid \varphi_{0}=i\right] \text { for } i \in \S .
$$

This is the usual conditional probability that the whole population eventually becomes extinct, given the type of the initial individual, and we write that $\boldsymbol{q}=\mathbb{P}\left[\lim _{n \rightarrow \infty}\left|\mathcal{Z}_{n}\right|=0 \mid \varphi_{0}\right]$ for short. The vector $\boldsymbol{q}$ is the minimal nonnegative solution of the fixed-point equation

$$
P(s)=s .
$$

This equation has at most two distinct solutions, $\mathbf{1}$ and $\boldsymbol{q} \leq \mathbf{1}$, if $M$ is irreducible, and potentially infinitely many solutions otherwise (see [10] and [14]).

The conditional partial extinction probability, given the initial type, is $\tilde{\boldsymbol{q}}=\left(\tilde{q}_{1}, \tilde{q}_{2}, \ldots\right)$, where

$$
\tilde{q}_{i}=\mathbb{P}\left[\text { for all } \ell: \lim _{n \rightarrow \infty} Z_{n \ell}=0 \mid \varphi_{0}=i\right] \text { for } i \in \& .
$$

In the irreducible case, Zucca [17] observed that $\lim _{n \rightarrow \infty} Z_{n \ell}=0$ for all types $\ell$ if and only if the limit is 0 for at least one type, regardless of the initial type.

The vector $\tilde{\boldsymbol{q}}$ is also a solution of (2.1). Indeed, by conditioning on the progeny of the initial individual and using the independence between individuals, we readily obtain, for any $i$,

$$
\begin{aligned}
\tilde{q}_{i} & =\mathbb{P}\left[\text { for all } \ell: \lim _{n \rightarrow \infty} Z_{n \ell}=0 \mid \varphi_{0}=i\right] \\
& =\sum_{j=\left(j_{1}, j_{2}, \ldots\right)} p_{i j} \prod_{k=1}^{\infty} \mathbb{P}\left[\text { for all } \ell: \lim _{n \rightarrow \infty} Z_{n \ell}=0 \mid \varphi_{0}=k\right]^{j_{k}} \\
& =P_{i}(\tilde{\boldsymbol{q}}) .
\end{aligned}
$$

When the set of types is finite, global and partial extinction are equivalent, but this is not necessarily the case when the set of types is infinite. By Fatou's lemma,

$$
\lim _{n \rightarrow \infty}\left|Z_{n}\right|=\lim _{n \rightarrow \infty} \sum_{\ell=1}^{\infty} Z_{n \ell} \geq \sum_{\ell=1}^{\infty} \lim _{n \rightarrow \infty} Z_{n \ell},
$$


so that

$$
\mathbb{P}\left[\lim _{n \rightarrow \infty}\left|Z_{n}\right|=0 \mid \varphi_{0}=i\right] \leq \mathbb{P}\left[\text { for all } \ell: \lim _{n \rightarrow \infty} Z_{n \ell}=0 \mid \varphi_{0}=i\right]
$$

for $i, \ell \in \&$ and $\mathbf{0} \leq \boldsymbol{q} \leq \tilde{\boldsymbol{q}} \leq \mathbf{1}$.

As the vectors $\boldsymbol{q}, \tilde{\boldsymbol{q}}$, and $\mathbf{1}$ are all solutions of (2.1) and since there are at most two distinct solutions in the irreducible case, the following lemma is immediate.

Lemma 2.1. If $M$ is irreducible, and $\tilde{\boldsymbol{q}}<\mathbf{1}$, then $\boldsymbol{q}=\tilde{\boldsymbol{q}}$.

In the irreducible case, $\boldsymbol{q}=\tilde{\boldsymbol{q}}<\mathbf{1}$ is equivalent to strong local survival in the terminology of branching random walks and, although it is expressed differently, this sufficient condition is observed in [2] with the assumption that $M$ is tridiagonal, and in [17] for the general case. When $M$ is reducible, it is possible to have $\boldsymbol{q}<\tilde{\boldsymbol{q}}<\mathbf{1}$, and we give an example at the end of Section 5 .

\section{Computational aspects}

In this section we develop iterative methods to compute the extinction probability vectors $\boldsymbol{q}$ and $\tilde{\boldsymbol{q}}$. The procedures apply to both irreducible and reducible processes. The underlying idea is to compute approximations of the infinite vectors $\boldsymbol{q}$ and $\tilde{\boldsymbol{q}}$ by solving finite systems of equations in such a way that the successive approximations have probabilistic interpretations: for $\boldsymbol{q}$, we use a time-truncation argument, and, for $\tilde{\boldsymbol{q}}$, we use a space-truncation argument.

\subsection{Global extinction probability}

Denote by $N_{\mathrm{e}}$ the generation number when the process becomes extinct. Clearly, $\boldsymbol{q}=$ $\mathbb{P}\left[N_{\mathrm{e}}<\infty \mid \varphi_{0}\right]$. Let $T_{k}=\{k+1, k+2, \ldots\}$ be the set of types strictly greater than $k$, and define the first passage time $\tau_{k}=\inf \left\{n: \sum_{\ell \in T_{k}} Z_{n \ell}>0\right\}$ for $k \geq 0$. This is the first generation when an individual of any type in $T_{k}$ is born. Furthermore, define

$$
q_{i}^{(k)}=\mathbb{P}\left[N_{\mathrm{e}}<\tau_{k} \mid \varphi_{0}=i\right],
$$

the conditional probability that the process eventually becomes extinct before the birth of any individual of a type in $T_{k}$, given that the initial individual has type $i$, and $\boldsymbol{q}^{(k)}=\left(q_{1}^{(k)}, q_{2}^{(k)}, \ldots\right)$.

Lemma 3.1. The sequence $\left\{\boldsymbol{q}^{(k)}\right\}_{k \geq 0}$ is monotonically increasing and converges pointwise to the global extinction probability vector $\boldsymbol{q}$.

Proof. Clearly, $T_{k} \supset T_{k+1}$ for all $k$, and $\tau_{k} \leq \tau_{k+1}$, so that $\left[N_{\mathrm{e}}<\tau_{k}\right] \subseteq\left[N_{\mathrm{e}}<\tau_{k+1}\right]$, and $\boldsymbol{q}^{(k)} \leq \boldsymbol{q}^{(k+1)}$. Therefore, for any $i$,

$$
\begin{aligned}
\lim _{k \rightarrow \infty} q_{i}^{(k)} & =\lim _{k \rightarrow \infty} \mathbb{P}\left[N_{\mathrm{e}}<\tau_{k} \mid \varphi_{0}=i\right] \\
& =\mathbb{P}\left[N_{\mathrm{e}}<\lim _{k \rightarrow \infty} \tau_{k} \mid \varphi_{0}=i\right] \\
& =\mathbb{P}\left[N_{\mathrm{e}}<\infty \mid \varphi_{0}=i\right] \\
& =q_{i},
\end{aligned}
$$

which completes the proof.

By definition, $q_{i}^{(k)}=0$ for all $i \in T_{k}$. Consequently,

$$
\boldsymbol{q}^{(0)}=(0,0, \ldots), \quad \boldsymbol{q}^{(k)}=\left(q_{1}^{(k)}, \ldots, q_{k}^{(k)}, 0,0, \ldots\right) \quad \text { for } k \geq 1 .
$$


Thus, at the $k$ th iteration we only need to compute the finite vector $\boldsymbol{w}^{(k)}=\left(q_{1}^{(k)}, \ldots, q_{k}^{(k)}\right)$, which we do as follows. Consider a branching process $\left\{\mathcal{W}_{n}^{(k)}\right\}_{n \in \mathbb{N}}$ which evolves like $\left\{\mathcal{Z}_{n}\right\}$ under taboo of the types in $T_{k}$. The taboo progeny distribution $f_{i j}^{(k)}$ associated with types $i \in\{1, \ldots, k\}$ in $\left\{W_{n}^{(k)}\right\}$ is defined as

$$
f_{i\left(j_{1}, \ldots, j_{k}\right)}^{(k)}=p_{i\left(j_{1}, \ldots, j_{k}, 0,0, \ldots\right)} .
$$

If the process is irreducible then $\sum_{\boldsymbol{j} \in \mathbb{N}^{k}} f_{i\left(j_{1}, \ldots, j_{k}\right)}^{(k)} \leq 1$ for $1 \leq i \leq k$ with at least one strict inequality, and we need to add an absorbing state $\Delta$ to the state space $\mathbb{N}^{k}$ of $\left\{\boldsymbol{W}_{n}^{(k)}\right\}$ to account for the missing probability mass. Obviously, absorption in $\Delta$ precludes extinction, and $\boldsymbol{w}^{(k)}$ is the vector of probability that $\left\{W_{n}^{(k)}\right\}$ becomes extinct before being absorbed in $\Delta$, given the type of the initial individual. Consequently, $\boldsymbol{w}^{(k)}$ is the minimal nonnegative solution of the finite system of equations

$$
s_{i}=F_{i}^{(k)}\left(s_{1}, s_{2}, \ldots, s_{k}\right) \quad \text { for } 1 \leq i \leq k,
$$

where $F_{i}^{(k)}(s)=P_{i}\left(s_{1}, \ldots, s_{k}, 0,0 \ldots\right)$ is the probability generating function of the distribution $f_{i j}^{(k)}$.

One procedure to compute $\boldsymbol{w}^{(k)}$ is by linear functional iteration, using the fixed-point equation (3.1). In that case, we easily verify that, for any $k \geq 1$, the sequence $\left\{\boldsymbol{w}^{(k, n)}=\left(w_{1}^{(k, n)}, \ldots\right.\right.$, $\left.\left.w_{k}^{(k, n)}\right)\right\}_{n \geq 0}$, recursively defined as

$$
w_{i}^{(k, n)}=F_{i}^{(k)}\left(w_{1}^{(k, n-1)}, \ldots, w_{k}^{(k, n-1)}\right) \quad \text { for } 1 \leq i \leq k, n \geq 1,
$$

with $\boldsymbol{w}^{(k, 0)}=(0,0 \ldots, 0)$, satisfies

$$
\boldsymbol{w}^{(k, n)}=\mathbb{P}\left[N_{\mathrm{e}}<\tau_{k} \text { and } N_{\mathrm{e}} \leq n \mid \varphi_{0}\right],
$$

and is therefore monotonically increasing to $\boldsymbol{w}^{(k)}$. In practice, we would terminate the functional iteration for a given $k$ when $\left\|\boldsymbol{w}^{(k, n+1)}-\boldsymbol{w}^{(k, n)}\right\|$ becomes sufficiently small.

\subsection{Partial extinction probability}

Here we associate to the branching process $\left\{Z_{n}\right\}$ a family of processes $\left\{Z_{n}^{(k)}=\left(Z_{n 1}^{(k)}\right.\right.$, $\left.\left.Z_{n 2}^{(k)}, \ldots\right)\right\}_{n \in \mathbb{N}}$ for $k \geq 0$ obtained as follows. For a given $k$, we count neither the individuals of types in $T_{k}$, nor any of their descendants, whatever their types. It is as if all individuals of types in $T_{k}$ became sterile. Define $\tilde{\boldsymbol{q}}^{(k)}$ to be the global extinction probability vector of the process $\left\{Z_{n}^{(k)}\right\}$.

Lemma 3.2. The sequence of vectors $\left\{\tilde{\boldsymbol{q}}^{(k)}\right\}_{k \geq 0}$ is monotonically decreasing and converges pointwise to the partial extinction probability vector $\tilde{\boldsymbol{q}}$.

Proof. Obviously,

$$
\begin{aligned}
Z_{n}^{(k)} & =\left(Z_{n 1}^{(k)}, Z_{n 2}^{(k)}, \ldots, Z_{n k}^{(k)}, 0,0,0, \ldots\right) \\
& \leq\left(Z_{n 1}^{(k+1)}, Z_{n 2}^{(k+1)}, \ldots, Z_{n k}^{(k+1)}, Z_{n(k+1)}^{(k+1)}, 0,0, \ldots\right) \text { a.s. } \\
& =Z_{n}^{(k+1)}
\end{aligned}
$$

so that, for fixed $n$ and $k \rightarrow \infty, \mathbb{Z}_{n}^{(k)}$ monotonically converges to $\mathbb{Z}_{n}$. Furthermore,

$$
\lim _{n \rightarrow \infty}\left|\mathcal{Z}_{n}^{(k)}\right| \leq \lim _{n \rightarrow \infty}\left|\mathcal{Z}_{n}^{(k+1)}\right|
$$

so that $\tilde{\boldsymbol{q}}^{(k)} \geq \tilde{\boldsymbol{q}}^{(k+1)}$ for $k \geq 0$. 
Let $B_{\ell}^{(k)}=\left[\lim _{n \rightarrow \infty} Z_{n \ell}^{(k)}=0\right]$ be the event that type $\ell$ of $\left\{Z_{n}^{(k)}\right\}$ eventually becomes extinct, and let $A^{(k)}=\bigcap_{\ell \geq 1} B_{\ell}^{(k)}$ be the event that all types of $\left\{\mathcal{Z}_{n}^{(k)}\right\}$ eventually become extinct. We have

$$
\tilde{\boldsymbol{q}}^{(k)}=\mathbb{P}\left[\lim _{n \rightarrow \infty}\left|Z_{n}^{(k)}\right|=0 \mid \varphi_{0}\right]=\mathbb{P}\left[A^{(k)} \mid \varphi_{0}\right],
$$

since $\left|Z_{n}^{(k)}\right|=\sum_{\ell} Z_{n, \ell}^{(k)}$ contains only finitely many nonzero terms. Furthermore, $B_{\ell}^{(k+1)} \subseteq$ $B_{\ell}^{(k)}$, so that $A^{(k+1)} \subseteq A^{(k)}$, and

$$
A^{(k)} \searrow A^{(\infty)}=\bigcap_{\ell \geq 1}\left[\lim _{n \rightarrow \infty} Z_{n \ell}=0\right] .
$$

Therefore,

$$
\lim _{k \rightarrow \infty} \tilde{\boldsymbol{q}}^{(k)}=\mathbb{P}\left[A_{\infty} \mid \varphi_{0}\right]=\mathbb{P}\left[\text { for all } \ell: \lim _{n \rightarrow \infty} Z_{n \ell}=0 \mid \varphi_{0}\right]=\tilde{\boldsymbol{q}}
$$

which completes the proof.

By the definition of $\left\{\mathcal{Z}_{n}^{(k)}\right\}, \tilde{q}_{i}^{(k)}=1$ for all $i \in T_{k}$, and so

$$
\tilde{\boldsymbol{q}}^{(0)}=(1,1, \ldots), \quad \tilde{\boldsymbol{q}}^{(k)}=\left(\tilde{q}_{1}^{(k)}, \ldots, \tilde{q}_{k}^{(k)}, 1,1, \ldots\right) \quad \text { for } k \geq 1 .
$$

To compute the finite vector $\widetilde{\boldsymbol{w}}^{(k)}=\left(\tilde{q}_{1}^{(k)}, \ldots, \tilde{q}_{k}^{(k)}\right)$, we may interpret $\left\{\mathcal{Z}_{n}^{(k)}\right\}$ as a GaltonWatson process with finitely many types and progeny distribution $\tilde{f}_{i j}^{(k)}$ defined as

$$
\tilde{f}_{i\left(j_{1}, \ldots, j_{k}\right)}^{(k)}=\sum_{j_{k+1}, j_{k+2}, \ldots \geq 0} p_{i\left(j_{1}, \ldots, j_{k}, j_{k+1}, j_{k+2}, \ldots\right)},
$$

and $\widetilde{\boldsymbol{w}}^{(k)}$ is the minimal nonnegative solution of the finite system of equations

$$
s_{i}=\widetilde{F}_{i}^{(k)}\left(s_{1}, s_{2}, \ldots, s_{k}\right) \text { for } 1 \leq i \leq k,
$$

where $\widetilde{F}_{i}^{(k)}(s)=P_{i}\left(s_{1}, \ldots, s_{k}, 1,1, \ldots\right)$ is the probability generating function of the distribution $\tilde{f}_{i j}^{(k)}$. This equation may be solved by functional iteration, as explained at the end of Subsection 3.1.

\section{Extinction criteria}

When the number of types is finite and $M$ is irreducible, it is well known that

$$
\begin{array}{ll}
\boldsymbol{q}<\mathbf{1} & \text { if } \operatorname{sp}(M)>1, \\
\boldsymbol{q}=\mathbf{1} & \text { if } \operatorname{sp}(M) \leq 1
\end{array}
$$

If $M$ is reducible then

$$
\boldsymbol{q} \supsetneqq \mathbf{1} \text { if and only if } \operatorname{sp}(M)>1,
$$

where we write $v \supsetneqq \mathbf{1}$ to indicate that $v_{i} \leq 1$ for all $i$, with at least one strict inequality. Indeed, if $M$ is reducible, there may exist some type (but not all) from which partial extinction is almost sure even if $\operatorname{sp}(M)>1$; see [4].

We expect that, in the infinite countable case, some analogue of $\operatorname{sp}(M)$ also plays a role in determining if extinction occurs a.s. or not. This is the case for partial extinction, but not necessarily for global extinction. 


\subsection{Partial extinction $-M$ irreducible}

We denote by $\widetilde{M}^{(k)}$ the mean progeny matrix of the process $\left\{Z_{n}^{(k)}\right\}$ defined in Section 3.2, and by $M^{(k)}$ the $k \times k$ north-west truncations of the infinite matrix $M$. As we do not count individuals with types in $T_{k}, \widetilde{M}^{(k)}$ is given by

$$
\tilde{M}^{(k)}=\left[\begin{array}{c|c}
M^{(k)} & 0 \\
\hline 0 & 0
\end{array}\right],
$$

and it is clear that $\tilde{\boldsymbol{q}}^{(k)}=\left(\tilde{q}_{1}^{(k)}, \ldots, \tilde{q}_{k}^{(k)}, 1,1, \ldots\right)=\mathbf{1}$ if $\operatorname{sp}\left(M^{(k)}\right) \leq 1$; otherwise, $\tilde{\boldsymbol{q}}^{(k)} \supsetneqq \mathbf{1}$, with $\left(\tilde{q}_{1}^{(k)}, \ldots, \tilde{q}_{k}^{(k)}\right)<\mathbf{1}$ if $M^{(k)}$ is irreducible.

We assume in this subsection that $M$ is irreducible. The convergence norm of $M$ is defined as follows. Let $R$ be the convergence radius of the power series $\sum_{k \geq 0} r^{k}\left(M^{k}\right)_{i j}$, which does not depend on $i$ and $j$. The convergence norm $v$ of $M$ is

$$
v=R^{-1}=\lim _{k \rightarrow \infty}\left\{\left(M^{k}\right)_{i j}\right\}^{1 / k}
$$

it is also the smallest value such that there exists a nonnegative vector $\boldsymbol{x}$ satisfying $\boldsymbol{x} M \leq v \boldsymbol{x}$; see [13, Definition 6.3]. Note that the convergence norm of a finite matrix is equal to its spectral radius.

If we assume that all but at most a finite number of truncations $M^{(k)}$ are irreducible then, by [13, Theorem 6.8], the sequence $\left\{\operatorname{sp}\left(M^{(k)}\right)\right\}$ is nondecreasing for sufficiently large $k$ and $\lim _{k \rightarrow \infty} \operatorname{sp}\left(M^{(k)}\right)=v$, and we can immediately show the following property.

Proposition 4.1. Assume that all but at most a finite number of truncations $M^{(k)}$ are irreducible. The partial extinction probability vector $\tilde{\boldsymbol{q}}$ is such that $\tilde{\boldsymbol{q}}<\mathbf{1}$ if and only if $v>1$.

Proof. If $v>1$, as $\operatorname{sp}\left(M^{(k)}\right) \nearrow v$, there exists some $k$ such that $\operatorname{sp}\left(M^{(k)}\right)>1$ and such that $M^{(k)}$ is irreducible. Thus, $\left(\tilde{q}_{1}^{(k)}, \ldots, \tilde{q}_{k}^{(k)}\right)<\mathbf{1}$ and, since $\tilde{\boldsymbol{q}}^{(k)} \searrow \tilde{\boldsymbol{q}}$ by Proposition 3.2, $\tilde{\boldsymbol{q}}<\mathbf{1}$ in the limit.

If $v \leq 1$ then, for all $k, \operatorname{sp}\left(M^{(k)}\right) \leq 1$ and $\tilde{\boldsymbol{q}}^{(k)}=\mathbf{1}$, which implies that $\tilde{\boldsymbol{q}}=\mathbf{1}$.

This was also observed by Gantert et al. [2] and Müller [11], who used different arguments.

\subsection{Partial extinction $-M$ reducible}

Let us assume now that the matrix $M$ is reducible. The sequence $\left\{\operatorname{sp}\left(M^{(k)}\right)\right\}$ is still nondecreasing, but its limit might not be the convergence norm of $M$. Let $\bar{v} \in[0, \infty]$ denote the limit. The proof of the proposition below is very similar to that of Proposition 4.1 and is omitted.

Proposition 4.2. The partial extinction probability vector $\tilde{\boldsymbol{q}}$ is such that $\tilde{\boldsymbol{q}}=\mathbf{1}$ if and only if $\bar{v} \leq 1$, and $\tilde{\boldsymbol{q}} \supsetneqq \mathbf{1}$ otherwise.

In other words, there exists at least one type $i$ such that $\tilde{q}_{i}<1$ if and only if $\bar{v}>1$. The next question is, for which $i$ does the inequality $\tilde{q}_{i}<1$ hold? We give below a necessary and sufficient condition for $\tilde{q}_{i}$ to be strictly less than 1 .

We write $i \rightarrow j$ when type $i$ has a positive probability to generate an individual of type $j$ in a subsequent generation, that is, if there exists $n \geq 1$ such that $\left(M^{n}\right)_{i j}>0$. We define equivalent classes $C_{1}, C_{2}, \ldots$ such that, for each $k$, if $i \in C_{k}$ then $j \in C_{k}$ if and only if $i \rightarrow j$ and $j \rightarrow i$ for all $j$. This induces a partition of the set of types $\delta$ and we write that $C_{k} \rightarrow C_{\ell}$ when there exist $i \in C_{k}$ and $j \in C_{\ell}$ such that $i \rightarrow j$. We denote by $M_{k}$ the irreducible mean 
progeny matrix restricted to types in $C_{k}$, that is, $M_{k}=\left(M_{i j}\right)_{i, j \in C_{k}}$, and by $v_{k}$ the convergence norm of $M_{k}$.

Proposition 4.3. If $i$ is a type in $C_{k}$ then the partial extinction probability $\tilde{q}_{i}$ is strictly less than 1 if and only if $v_{k}>1$ or there exists a class $C_{\ell}$ such that $C_{k} \rightarrow C_{\ell}$ and $v_{\ell}>1$.

Proof. Let $i \in C_{k}$, and assume that $v_{k}>1$. Then, by Proposition 4.1, the probability that every type in $C_{k}$ eventually becomes extinct, given that the initial type is in $C_{k}$, is strictly less than 1 ; hence, $\tilde{q}_{i}<1$.

Now assume that $i \in C_{k}$, and that there exists a class $C_{\ell}$ such that $C_{k} \rightarrow C_{\ell}$ and $\nu_{\ell}>1$. Then, $i \rightarrow j$ for all $j \in C_{\ell}$, that is, there is a positive probability that type $i$ has type $j \in C_{\ell}$ among its descendants; moreover, since $v_{\ell}>1$, starting from any $j \in C_{\ell}$, the probability that every type in $C_{\ell}$ eventually becomes extinct is strictly less than 1 by Proposition 4.1 . We thus obtain $\tilde{q}_{i}<1$.

If $v_{k} \leq 1$ and there is no class $C_{\ell}$ such that $C_{k} \rightarrow C_{\ell}$ and $v_{\ell}>1$, then all classes $C_{\ell}$ such that $C_{k} \rightarrow C_{\ell}$ satisfy $v_{\ell} \leq 1$. In other words, by Proposition 4.1, all the descendants of type $i$ will generate a process which partially becomes extinct with probability 1 . So partial extinction is almost sure if the process is initiated by type $i$, and we have $\tilde{q}_{i}=1$.

\subsection{Global extinction- $M$ irreducible}

We assume again that $M$ is irreducible. By Lemma 2.1 and Proposition 4.1, we know that if $v>1$ then $\boldsymbol{q}=\tilde{\boldsymbol{q}}<\mathbf{1}$, and if $v \leq 1$ then $\boldsymbol{q} \leq \tilde{\boldsymbol{q}}=\mathbf{1}$. One problem which remains is to determine additional conditions that guarantee $\boldsymbol{q}=\tilde{\boldsymbol{q}}=\mathbf{1}$.

The most precise answers are conditioned on the dichotomy property, which states that, with probability 1, the population either becomes extinct or drifts to $\infty$; see [3]. In the finite case, this follows under very mild conditions but it is more problematic if the number of types is infinite. In particular, Tetzlaff [15, Condition 2.1 and the proof of Proposition 2.2] gave the following sufficient condition for the dichotomy property to hold: it suffices that, for all $k \geq 1$, there exists an index $m_{k}$ and a real number $d_{k}>0$ such that

$$
\inf _{i} \mathbb{P}\left[\left|Z_{m_{k}}\right|=0\left|\varphi_{0}=i, 1 \leq\right| Z_{1} \mid \leq k\right] \geq d_{k} .
$$

This indicates that there is a positive, and bounded away from 0 , probability for the population to become extinct. The next property is proved in [15].

Proposition 4.4. Assume that the dichotomy property holds. If the limit $\lim _{\inf } \rightarrow \infty\left(M^{n} \mathbf{1}\right)$ is finite then $\boldsymbol{q}=\mathbf{1}$.

A direct consequence, which brings the convergence norm back into the picture, is the following.

Proposition 4.5. Assume that the dichotomy property holds. If there exist $\lambda \leq 1$ and $\boldsymbol{x}>\boldsymbol{0}$ such that $\boldsymbol{x} \mathbf{1}<\infty$ and $\boldsymbol{x} M \leq \lambda \boldsymbol{x}$, then $\boldsymbol{q}=\mathbf{1}$.

Proof. Under the assumptions of the proposition, $\boldsymbol{x} M^{n} \mathbf{1} \leq \lambda^{n} \boldsymbol{x} \mathbf{1}$, which implies that $\lim _{n \rightarrow \infty} \boldsymbol{x} M^{n} \mathbf{1}<\infty$. Applying Fatou's lemma, we obtain $x \lim _{n \rightarrow \infty}\left(M^{n} \mathbf{1}\right)<\infty$, which leads to $\lim _{n \rightarrow \infty}\left(M^{n} \mathbf{1}\right)<\infty$ since $\boldsymbol{x}>\mathbf{0}$. Thus, by Proposition 4.4, the result follows.

If such a $\lambda$ exists, it is at least equal to $v$, and we remember that $v \leq 1$ is a necessary condition for $\boldsymbol{q}=\mathbf{1}$. The difference between $\lambda$ and $v$, and the additional constraint imposed by this proposition, is that the measure associated to $\lambda$ must be convergent, which is not necessarily the case with the measure associated with $v$. 


\subsection{Growth rate and extinction}

When the number of types is finite and the process is irreducible, the expected total population size increases, or decreases, asymptotically geometrically: independently of the initial type, $\mathbb{E}\left[\left|\mathcal{Z}_{n}\right|\right] \sim \rho^{n}$, where $\rho$ is the spectral radius of $M$. This is no longer the case when the number of types is infinite, and the evolution of $\mathbb{E}\left[\left|\mathcal{Z}_{n}\right|\right]$ may depend on the distribution of $\varphi_{0}$. Actually, it is possible for a process to become globally extinct a.s. while the expected population size increases without bounds. This we show below, and we give one example in the next section.

Assume that there exists a probability measure $\alpha_{1}$ such that $\alpha_{1} M \leq \lambda_{1} \alpha_{1}$ with $\lambda_{1}<1$, and a probability measure $\boldsymbol{\alpha}_{2}$ such that $\boldsymbol{\alpha}_{2} M \geq \lambda_{2} \boldsymbol{\alpha}_{2}$ with $\lambda_{2}>1$. If, in addition, the dichotomy property holds, then $\boldsymbol{q}=\mathbf{1}$ by Proposition 4.5.

If $\varphi_{0}$ has distribution $\boldsymbol{\alpha}_{1}$ then

$$
\mathbb{E}\left[\left|Z_{n}\right|\right]=\boldsymbol{\alpha}_{1} M^{n} \mathbf{1} \leq \lambda_{1}^{n},
$$

so that $\lim _{n \rightarrow \infty} \mathbb{E}\left[\left|\mathcal{Z}_{n}\right|\right]=0$. If $\varphi_{0}$ has distribution $\boldsymbol{\alpha}_{2}$ then, by a similar argument, $\lim _{n \rightarrow \infty} \mathbb{E}\left[\left|\mathcal{Z}_{n}\right|\right]=\infty$ and the extinction probability is equal to $\boldsymbol{\alpha}_{2} \boldsymbol{q}=1$.

\section{Illustration}

We illustrate the results of the previous sections with two examples, one for which $M$ is tridiagonal (and the process is irreducible) and one for which $M$ is superdiagonal (and the process is reducible).

\subsection{Two-sided branching random walk}

This example corresponds to a homogeneous branching random walk on the positive integers with a reflecting wall at $z=1$. The mean progeny matrix is

$$
M=\left[\begin{array}{ccccc}
b & c & & & \\
a & b & c & & \\
& a & b & c & \\
& & \ddots & \ddots & \ddots
\end{array}\right],
$$

where $a$ and $c$ are strictly positive, and $b$ is nonnegative.

Proposition 5.1. Assume that $M$ is as given in (5.1). Then, its convergence norm is $v=$ $b+2 \sqrt{a c}$, and there exists $\boldsymbol{x} \equiv \boldsymbol{x}(\lambda)>\mathbf{0}$ such that $\boldsymbol{x} M=\lambda \boldsymbol{x}$ for all $\lambda \geq \nu$. In addition, $\boldsymbol{x} \mathbf{1}<\infty$ if and only if $\lambda \in[v, a+b+c)$ and $a>c$.

The strictly positive and convergent invariant measure $\boldsymbol{x}$ is given by

$$
x_{k}= \begin{cases}\eta k\left(\frac{\sqrt{a c}}{a}\right)^{k} & \text { if } \lambda=\nu, \\ \eta\left\{\left(\frac{\lambda-b+\sqrt{\Delta}}{2 a}\right)^{k}-\left(\frac{\lambda-b-\sqrt{\Delta}}{2 a}\right)^{k}\right\} & \text { if } \lambda>v,\end{cases}
$$

for $k \geq 1$, where $\eta$ is an arbitrary constant and $\Delta=(b-\lambda)^{2}-4 a c$.

Proof. Let $M^{(k)}$ denote the $k \times k$ north-west truncations of $M$. Then, by a modification of [16, Theorem 1, Equation (9)] we obtain

$$
\operatorname{sp}\left(M^{(k)}\right)=\min _{\boldsymbol{u} \geq \mathbf{0}} \max _{1 \leq i \leq k}\left\{b+u_{i+1}+\frac{a c}{u_{i}}\right\} .
$$


Then, by [13, Theorem 6.8],

$$
v=\lim _{k \rightarrow \infty} \operatorname{sp}\left(M^{(k)}\right)=\min _{\boldsymbol{u} \geq \mathbf{0}} \sup _{i}\left\{b+u_{i+1}+\frac{a c}{u_{i}}\right\}=b+2 \sqrt{a c},
$$

with the last equality following from arguments analogous to those in the proof of [6, Theorem 3.2].

Now, for any $\boldsymbol{x}$ to satisfy $\boldsymbol{x} M=\lambda \boldsymbol{x}$, its elements have to satisfy the constraints

$$
\begin{gathered}
b x_{1}+a x_{2}=\lambda x_{1}, \\
a x_{k+1}+(b-\lambda) x_{k}+c x_{k-1}=0 \quad \text { for } k \geq 2 .
\end{gathered}
$$

Let $\Delta=(b-\lambda)^{2}-4 a c$. There are three cases, for each of which $\boldsymbol{x}$ takes a specific form (see [5, Chapter 20, Section 4.5]).

Case (i): $\Delta=0$. Then $\lambda=b \pm 2 \sqrt{a c}$ and, for $k \geq 1$,

$$
x_{k}=c_{1}\left(\frac{\lambda-b}{2 a}\right)^{k}+c_{2} k\left(\frac{\lambda-b}{2 a}\right)^{k},
$$

where $c_{1}$ and $c_{2}$ are constants. Substituting (5.5) into (5.4) gives $c_{1}=0$. To ensure that $x_{k}>0$ for all $k$, it is necessary that $\lambda>b$. Consequently, $\lambda=b+2 \sqrt{a c}$, and we obtain (5.2). For $\boldsymbol{x}$ to be convergent, we require that $\sqrt{a c} / a<1$ and, thus, $a>c$.

Case (ii): $\Delta>0$. Then $\lambda<b-2 \sqrt{a c}$ or $\lambda>b+2 \sqrt{a c}$ and, for $k \geq 1$,

$$
x_{k}=c_{3}\left(\frac{\lambda-b+\sqrt{\Delta}}{2 a}\right)^{k}+c_{4}\left(\frac{\lambda-b-\sqrt{\Delta}}{2 a}\right)^{k},
$$

where $c_{3}$ and $c_{4}$ are constants. Substituting (5.6) into (5.4) gives us $c_{3}=-c_{4}$, and (5.6) simplifies to (5.3). It is clear from (5.3) that $\boldsymbol{x}>\mathbf{0}$ if and only if $\lambda>b$. Thus, $\lambda>b+2 \sqrt{a c}$.

Finally, $\boldsymbol{x} \mathbf{1}<\infty$ if and only if $(\lambda-b+\sqrt{\Delta}) /(2 a)<1$; the latter being equivalent to $\lambda<2 a+b$ and $\lambda<a+b+c$. As $b+2 \sqrt{a c}<\lambda<2 a+b$, both $a<c$ and $a=c$ lead to a contradiction. Consequently, $a>c$.

Case (iii): $\Delta<0$. Then $b-2 \sqrt{a c}<\lambda<b+2 \sqrt{a c}$ and

$$
x_{k}=\left(\frac{c}{a}\right)^{k}\left(c_{5} \cos (k \phi)+c_{6} \sin (k \phi)\right),
$$

where $\phi=\arccos (b /(2 \sqrt{a c})), 0<\phi<\pi$, and $c_{5}$ and $c_{6}$ are constants.

Since we are looking for $\boldsymbol{x}>\mathbf{0}$, case (iii) is not feasible. Indeed, we can rewrite (5.7) as, for $k \geq 1, x_{k}=c_{7}(c / a)^{k} \cos \left(c_{8}+k \phi\right)$, where $0 \leq c_{8}<2 \pi$ and $c_{7}$ is arbitrary. It can be easily shown that there exists $k_{0}$ such that $\cos c_{8}$ and $\cos \left(c_{8}+k_{0} \phi\right)$ have different signs. This completes the proof of the proposition.

Among the progeny distributions that may be associated with the mean progeny matrix given in (5.1), we choose

$$
P_{i}(\boldsymbol{s})= \begin{cases}\frac{b}{t} s_{i}^{t}+\frac{c}{t} s_{i+1}^{t}+1-\frac{b+c}{t} & \text { for } i=1, \\ \frac{a}{u} s_{i-1}^{u}+\frac{b}{u} s_{i}^{u}+\frac{c}{u} s_{i+1}^{u}+1-\frac{a+b+c}{u} & \text { for } i \geq 2,\end{cases}
$$



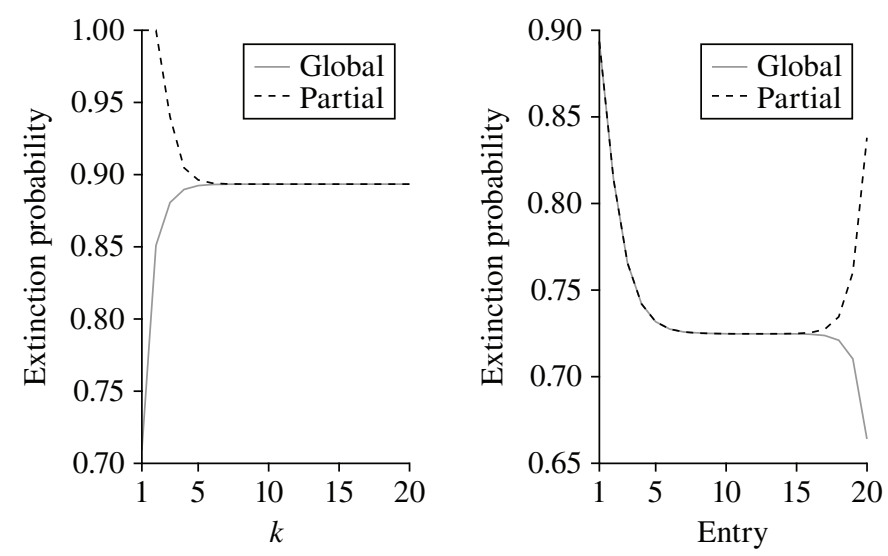

FIGURE 1: Case (i) with $a=b=\frac{1}{2}$ and $c=\frac{1}{3}$. Left: the values of $q_{1}^{(k)}$ (solid line) and of $\tilde{q}_{1}^{(k)}$ (dashed line). Right: the first entries of $\boldsymbol{q}^{(20)}$ (solid line) and $\tilde{\boldsymbol{q}}^{(20)}$ (dashed line).

where $t=\lceil b+c\rceil+1$ and $u=\lceil a+b+c\rceil+1$. By varying $a, b$, and $c$, we shall cover the three possible cases $\boldsymbol{q}=\tilde{\boldsymbol{q}}<\mathbf{1}, \boldsymbol{q}=\tilde{\boldsymbol{q}}=\mathbf{1}$, and $\boldsymbol{q}<\tilde{\boldsymbol{q}}=\mathbf{1}$.

Case (i): $\boldsymbol{q}=\tilde{\boldsymbol{q}}<\mathbf{1}$. Take $a=b=\frac{1}{2}$ and $c=\frac{1}{3}$. With these values, $v=1.28>1$ and $\boldsymbol{q}=\tilde{\boldsymbol{q}}<\mathbf{1}$ by Lemma 2.1 and Proposition 4.1 .

We illustrate in Figure 1 the convergence of the sequences $\left\{\boldsymbol{q}^{(k)}\right\}$ and $\left\{\tilde{\boldsymbol{q}}^{(k)}\right\}$. In the left-hand diagram, we $\operatorname{plot} q_{1}^{(k)}$ and $\tilde{q}_{1}^{(k)}$ for $k=1$ to 20 ; the two sequences rapidly converge to a common value approximately equal to 0.89 . In the right-hand diagram, we plot $q_{i}^{(20)}$ and $\tilde{q}_{i}^{(20)}$ for $i=1$ to 20; we observe that the first 15 entries are well approximated after 20 iterations but the next entries require more iterations because, for high values of $i$, the approximation process for $q_{i}$ and $\tilde{q}_{i}$ starts with a higher value of $k$.

A sequence $\left\{x_{k}\right\}_{k \geq 0}$ converges linearly to $x$ if there exists $0<\mu<1$ such that $\lim _{k \rightarrow \infty} \mid x-$ $x_{k+1}|/| x-x_{k} \mid=\mu$, and $\mu$ is called the convergence rate. Our numerical investigations indicate that the convergence of $q_{i}^{(k)}$, as well as that of $\tilde{q}_{i}^{(k)}$, is linear for fixed $i$. We give an example in Figure 2, where we plot the ratios $\left|q_{1}-q_{1}^{(k+1)}\right| /\left|q_{1}-q_{1}^{(k)}\right|$ and $\left|\tilde{q}_{1}-\tilde{q}_{1}^{(k+1)}\right| /\left|\tilde{q}_{1}-\tilde{q}_{1}^{(k)}\right|$; not knowing the value of either $q_{1}$ or $\tilde{q}_{1}$, we have used the values at the 20 th iteration. The two sequences are seen to converge linearly at the same rate $\mu=0.26$ approximately.

Case (ii): $\boldsymbol{q}=\tilde{\boldsymbol{q}}=\mathbf{1}$. Take $a=b=\frac{1}{2}$ and $c=\frac{1}{25}$. Here $a>c$ and, for any individual, the type of its descendants drifts over successive generations toward type 1 , the least prolific of types. The convergence norm is $v=0.78<1$, which implies that $\tilde{\boldsymbol{q}}=\mathbf{1}$. We shall conclude, from Proposition 5.1 and Proposition 4.5 (with $\lambda=v$ ), that $\boldsymbol{q}=\mathbf{1}$ as well, once we show that the dichotomy property holds. The progeny generating function is given by

$$
P_{i}(\boldsymbol{s})= \begin{cases}\frac{1}{4} s_{i}^{2}+\frac{1}{50} s_{i+1}^{2}+\frac{73}{100} & \text { for } i=1, \\ \frac{1}{6} s_{i-1}^{3}+\frac{1}{6} s_{i}^{3}+\frac{1}{75} s_{i+1}^{3}+\frac{49}{75} & \text { for } i \geq 2 .\end{cases}
$$

To verify that the dichotomy property holds, we use the sufficient condition (4.1). In view of (5.8) and (5.9), we observe that, for all $i, \mathbb{P}\left[\left|Z_{2}\right|=0\left|\varphi_{0}=i, 1 \leq\right| \mathcal{Z}_{1} \mid \leq k\right] \geq$ $\left(\min \left(\frac{73}{100}, \frac{49}{75}\right)\right)^{k}$, and we conclude that (4.1) is satisfied with $m_{k}=2$ and $d_{k}=\left(\frac{49}{75}\right)^{k}$. 


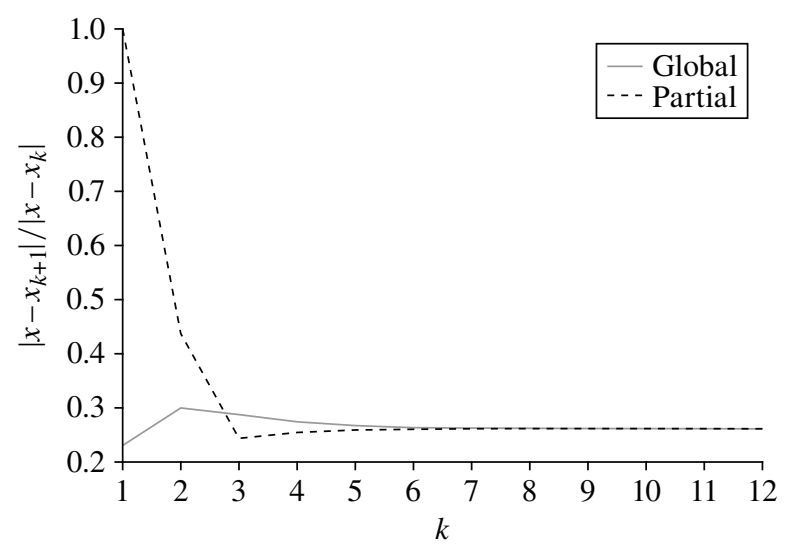

FIgURE 2: Case (i) with $a=b=\frac{1}{2}$ and $c=\frac{1}{3}$. Convergence rates of the sequences $q_{1}^{(k)}$ (solid line) and $\tilde{q}_{1}^{(k)}$ (dashed line).

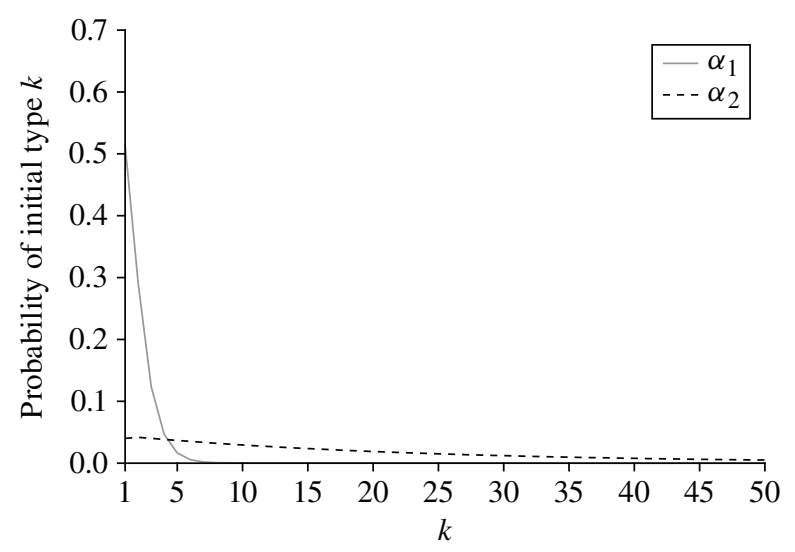

FIgURE 3: Case (ii) with $a=b=\frac{1}{2}$ and $c=\frac{1}{25}$. The first 50 components of the initial type distribution vector $\boldsymbol{\alpha}_{1}$, for which $\lim _{n} \mathbb{E}\left[\left|Z_{n}\right|\right]=0$, and of $\boldsymbol{\alpha}_{2}$, for which $\lim _{n} \mathbb{E}\left[\left|Z_{n}\right|\right]=\infty$.

To illustrate the observation made in Subsection 4.4 about the effects of the initial type's distribution, we take the parameters

$$
\lambda_{1}=v=0.78<1 \quad \text { and } \quad \lambda_{2}=1.02<1.04(=a+b+c) .
$$

By Proposition 5.1, there exist $\boldsymbol{\alpha}_{1}>0$ and $\boldsymbol{\alpha}_{2}>0$ such that $\boldsymbol{\alpha}_{1} M=\lambda_{1} \boldsymbol{\alpha}_{1}$ and $\boldsymbol{\alpha}_{1} \mathbf{1}=1$, and such that $\boldsymbol{\alpha}_{2} M=\lambda_{2} \boldsymbol{\alpha}_{2}$ and $\boldsymbol{\alpha}_{2} \mathbf{1}=1$.

If $\varphi_{0}$ has the distribution $\boldsymbol{\alpha}_{1}$ then $\lim _{n} \mathbb{E}\left[\left|\mathcal{Z}_{n}\right|\right]=0$; while if it has the distribution $\boldsymbol{\alpha}_{2}$ then $\lim _{n} \mathbb{E}\left[\left|\mathcal{Z}_{n}\right|\right]=\infty$. In both cases, extinction is with probability 1 . We plot in Figure 3 the first 50 components of $\boldsymbol{\alpha}_{1}$ and $\boldsymbol{\alpha}_{2}$. The difference between the two is that the distribution $\boldsymbol{\alpha}_{1}$ is concentrated on small types, so that the process has less chance of building a high population before its eventual extinction.

Case (iii): $\boldsymbol{q} \leq \tilde{\boldsymbol{q}}=\mathbf{1}$. Take $a=\frac{1}{25}$ and $b=c=\frac{1}{2}$. Here, $a<c$ and $v=0.78<1<$ $a+b+c$; thus, $\boldsymbol{q} \leq \tilde{\boldsymbol{q}}=\mathbf{1}$, but we do not know whether or not $\boldsymbol{q}=\mathbf{1}$. 

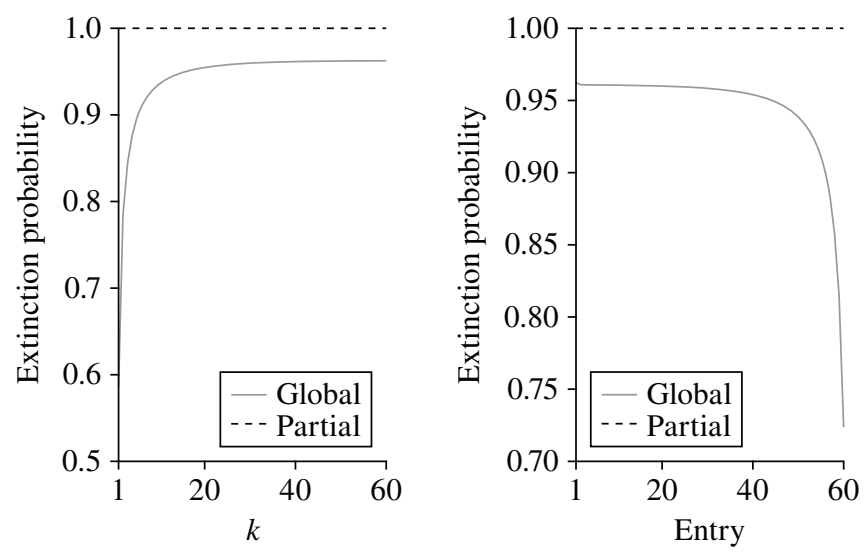

FIGURE 4: Case (iii) with $a=\frac{1}{25}$ and $b=c=\frac{1}{2}$. Left: the values of $q_{1}^{(k)}$ (solid line) and of $\tilde{q}_{1}^{(k)}$ (dashed line). Right: the first entries of $\boldsymbol{q}^{(60)}$ (solid line) and $\tilde{\boldsymbol{q}}^{(60)}$ (dashed line).

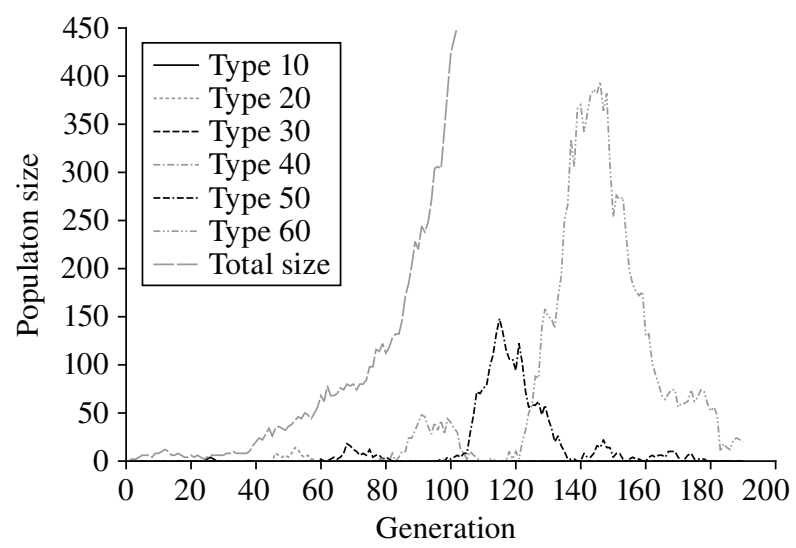

Figure 5: Case (iii) with $a=\frac{1}{25}$ and $b=c=\frac{1}{2}$. Simulation of the evolution of the population size in different types and of the total population size.

We show in the left-hand diagram of Figure 4 the values of $q_{1}^{(k)}$ and $\tilde{q}_{1}^{(k)}$ for $k=1$ to 60 . Judging from this, we conclude that $q_{1}<1=\tilde{q}_{1}$. In the right-hand diagram of that figure, we give $q_{i}^{(60)}$ and $\tilde{q}_{i}^{(60)}$ for $i=1$ to 60 .

To confirm that $\boldsymbol{q}<\tilde{\boldsymbol{q}}=\mathbf{1}$, we have simulated the branching process and we give one particular sample path in Figure 5: the whole population $\left|\mathcal{Z}_{n}\right|$ seems to grow without bounds, while individual types appear, grow in importance, and eventually disappear from the population.

\subsection{One-sided branching random walk}

Consider the mean progeny matrix with the structure

$$
M=\left[\begin{array}{ccccc}
b_{1} & c_{1} & & & \\
& b_{2} & c_{2} & & \\
& & b_{3} & c_{3} & \\
& & & \ddots & \ddots
\end{array}\right],
$$

where $b_{i} \geq 0$ and $c_{i}>0$ for all $i$. 

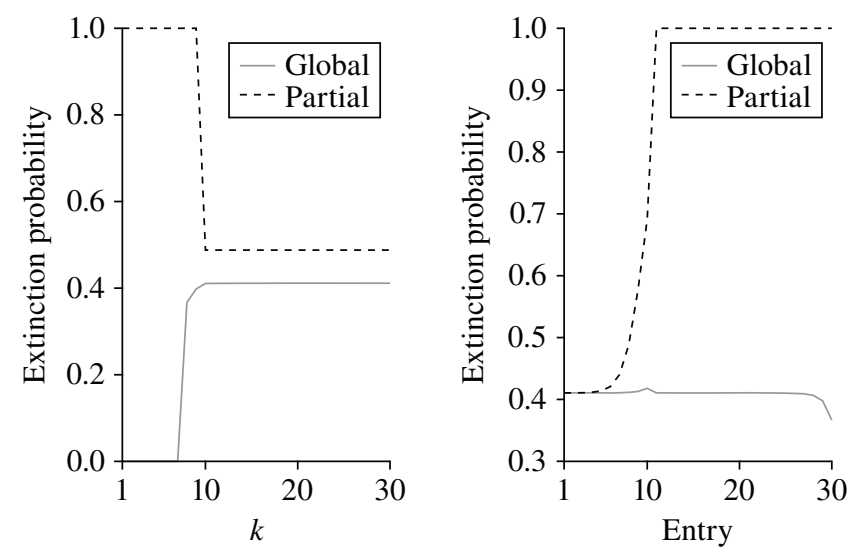

FIGURE 6: Left: the values of $q_{8}^{(k)}$ (solid line) and of $\tilde{q}_{8}^{(k)}$ (dashed line). Right: the first entries of $\boldsymbol{q}^{(30)}$ (solid line) and $\tilde{\boldsymbol{q}}^{(30)}$ (dashed line).

In this special case of a reducible mean progeny matrix we may associate another interpretation to the sequence $\left\{\tilde{\boldsymbol{q}}^{(k)}\right\}$. Let us define the local extinction of a specific type. This event is $E_{k}=\left[\lim _{n \rightarrow \infty} Z_{n k}=0\right]$, independently of the other types.

A moment of reflection shows that $E_{k} \equiv \bigcap_{\ell \leq k} E_{\ell}$ and, furthermore, that $\tilde{q}_{i}^{(k)}$ is the probability that type $k$ eventually becomes extinct, given that the process starts with a first individual of type $i$. This allows us to give another proof that $\tilde{\boldsymbol{q}}^{(k)} \geq \tilde{\boldsymbol{q}}^{(k+1)}$ and that the sequence converges to $\tilde{\boldsymbol{q}}$ :

$$
\lim _{k \rightarrow \infty} \tilde{\boldsymbol{q}}^{(k)}=\lim _{k \rightarrow \infty} \mathbb{P}\left[E_{k} \mid \varphi_{0}\right]=\lim _{k \rightarrow \infty} \mathbb{P}\left[\bigcap_{\ell \leq k} E_{\ell} \mid \varphi_{0}\right]=\mathbb{P}\left[\bigcap_{\ell \leq \infty} E_{\ell} \mid \varphi_{0}\right]=\tilde{\boldsymbol{q}} .
$$

In the reducible case, the equation $s=\boldsymbol{P}(\boldsymbol{s})$ may have more than two distinct solutions and, in particular, it is possible that $\boldsymbol{q}<\tilde{\boldsymbol{q}}<\mathbf{1}$, as we show with an example.

Take $b_{i}=0$ and $c_{i}=1.9$ for every $i$ except for $i=10$, where $b_{10}=1.6$ and $c_{10}=0.8$. That is, in general, type- $i(i \neq 10)$ individuals have only children of the next type and slightly less than two on average, while a type-10 individual is different. If it were not for type 10 , the whole population would behave as a supercritical process, with each type becoming extinct after one generation. Individuals of type 10 do reproduce themselves, in a supercritical fashion.

Assume that the progeny generating function is

$$
P_{i}(s)= \begin{cases}\frac{19}{30} s_{i+1}^{3}+\frac{11}{30} & \text { for } i \neq 10 \\ \frac{2}{5} s_{i}^{4}+\frac{1}{5} s_{i+1}^{4}+\frac{2}{5} & \text { for } i=10\end{cases}
$$

As the sequence $\left\{\operatorname{sp}\left(M^{(k)}\right)\right\}$ converges to $\bar{v}=1.6>1$, we know by Proposition 4.2 that $\tilde{\boldsymbol{q}} \supsetneqq \mathbf{1}$. Furthermore, Proposition 4.3 implies that $\tilde{q}_{i}<1$ for $1 \leq i \leq 10$, and $\tilde{q}_{i}=1$ for $i \geq 11$.

We show $\left\{q_{8}^{(k)}\right\}$ and $\left\{\tilde{q}_{8}^{(k)}\right\}$ in the left-hand diagram of Figure 6 and the plot clearly makes it appear that $q_{8}<\tilde{q}_{8}<1$. In the right-hand diagram, we give the values of $q_{i}^{(30)}$ and $\tilde{q}_{i}^{(30)}$ for $1 \leq i \leq 30$. For $i \geq 11$, local extinction has probability 1 since every type exists for one generation only, and the global extinction probability, at least if $i$ is sufficiently smaller than 30 , is close to 0.41 , the extinction probability of a single-type branching process with progeny 
generating function

$$
P(s)=\frac{19}{30} s^{3}+\frac{11}{30} .
$$

We thus see that if extinction happens in the single-type process, then it does so in a few generations.

\section{Acknowledgements}

All three authors thank the Ministère de la Communauté française de Belgique for funding this research through the ARC grant AUWB-08/13-ULB 5. The first and third authors also acknowledge the financial support of the Australian Research Council through the Discovery Grant DP110101663.

\section{References}

[1] Bertacchi, D. And ZuCCA, F. (2009). Approximating critical parameters of branching random walks. J. Appl. Prob. 46, 463-478.

[2] Gantert, N., Müller, S., Popov, S. and Vachkovskaia, M. (2010). Survival of branching random walks in random environment. J. Theoret. Prob. 23, 1002-1014.

[3] Harris, T. E. (1963). The Theory of Branching Processes. Springer, Berlin.

[4] Hautphenne, S. (2012). Extinction probabilities of supercritical decomposable branching processes. J. Appl. Prob. 49, 639-651.

[5] Korn, G. A. And Korn, T. M. (1961). Mathematical Handbook for Scientists and Engineers: Definition, Theorems, and Formulas for Reference and Review. McGraw-Hill, New York.

[6] Latouche, G., Nguyen, G. T. and Taylor, P. G. (2011). Queues with boundary assistance: The effects of truncation. Queueing Systems 69, 175-197.

[7] Mode, C. J. (1971). Multi-Type Branching Processes: Theory and Application. Elsevier, New York.

[8] Moy, S.-T. C. (1967). Ergodic properties of expectation matrices of a branching process with countably many types. J. Math. Mech. 16, 1207-1225.

[9] Moy, S.-T. C. (1967). Extensions of a limit theorem of Everett, Ulam and Harris on multitype branching processes to a branching process with countably many types. Ann. Math. Statist. 38, 992-999.

[10] Moyal, J. E. (1962). Multiplicative population chains. Proc. R. Soc. London A. 266, 518-526.

[11] MülleR, S. (2008). A criterion for transience of multidimensional branching random walk in random environment. Electron. J. Prob. 13, 1189-1202.

[12] Sagitov, S. (2013). Linear-fractional branching processes with countably many types. Stoch. Process. Appl. 123, 2940-2956.

[13] Seneta, E. (1981). Nonnegative Matrices and Markov Chains, 2nd edn. Springer, New York.

[14] Spataru, A. (1989). Properties of branching processes with denumerably many types. Romanian J. Pure Appl. Math. 34, 747-759.

[15] Tetzlaff, G. T. (2003). Criticality in discrete time branching processes with not uniformly bounded types. Rev. Mat. Apl. 24, 25-36.

[16] van Doorn, E. A., van Foreest, N. D. and Zeifman, A. I. (2009). Representations for the extreme zeros of orthogonal polynomials. J. Comput. Appl. Math. 233, 847-851.

[17] ZuCCA, F. (2011). Survival, extinction and approximation of discrete-time branching random walks. J. Statist. Phys. 142, 726-753. 